\title{
Fundamental and Interdisciplinary Questions Drive the Scientific Exploration of Mercury
}

Lead Author: Steven A. Hauck, II, Case Western Reserve University, hauck@case.edu, (216) 368-3675

Co-authors:

David Blewett (JHU APL)

Paul K. Byrne (NCSU)

Nancy L. Chabot (JHU APL)

Carolyn M. Ernst (JHU APL)

Catherine L. Johnson (UBC \& PSI)

Erwan Mazarico (NASA GSFC)

Jim M. Raines (Michigan)

Kathleen E. Vander Kaaden (Jacobs/NASA JSC)

Ronald J. Vervack, Jr. (JHU APL)

Ariel Deutsch (Brown)

Gina DiBraccio (NASA GSFC)

Suzanne Imber (Leicester)

Christian Klimczak (Georgia)

Gangkai Poh (NASA GSFC/Catholic Univ.) 


\section{Executive Summary}

The planet Mercury is an end-member among the bodies of the inner solar system in terms of its formation, composition, evolution, and interaction between the sun and space environment. Mercury is also the least explored of the major terrestrial bodies of the inner solar system. Starting at the Mercury 2018 Workshop, followed by a community survey in the summer of 2019 described in a white paper to NASA Planetary Science leadership, the Mercury community has engaged in a process of identifying high-priority science questions. Asking questions is the fundamental and underlying process by which we advance science and exploration. This ongoing scientific and goal-setting conversation has provided a set of over-arching and, crucially, interconnected questions vital for the exploration and understanding of Mercury and the solar system. These questions are:

- How did Mercury form?

- How did Mercury differentiate and acquire its interior structure?

- What is the history of Mercury's magnetic field and its generation?

- How do Mercury's surface and interior reflect the evolution of the planet?

- What is the nature of the complex interactions among Mercury's external drivers and the planet's magnetosphere, exosphere, surface, and interior?

- What are the origin, history, and inventory of Mercury's volatiles?

The modern day Mercury is the result of a system of interconnected processes from the planet's center to beyond its magnetosphere that have operated both consecutively and concurrently. Through a rich set of research and analysis opportunities, ground-based observations, and exploration missions, the exploration of Mercury can unlock a new understanding of planetary formation, evolution, and interactions with host stars in our own solar system and beyond.

\section{Background}

Past exploration of Mercury has shown it to be unlike any other planetary body explored in the solar system. As the innermost planet, Mercury may also represent a valuable analog environment to the large numbers of exoplanets that have been discovered proximal to their host stars. Visited by only two spacecraft to date-NASA's Mariner 10 with three flybys in 1974-1975 and MESSENGER with a series of three flybys beginning in 2008 and an orbital campaign from 20112015-Mercury is also the least explored of the major terrestrial bodies of the inner solar system. The ESA/JAXA BepiColombo dual orbiters are set to arrive in orbit about Mercury in late 2025, positioned to return new remote sensing and in situ datasets about the planet and its surrounding space environment.

MESSENGER's orbital reconnaissance of Mercury provided the first global measurements of the near-space environment, surface, and interior of the planet. The data that MESSENGER returned enabled understanding of the planet's general internal structure, surface composition, extensive volcanic and tectonic history, and coupling between the dynamic exosphere and the local space environment. Discoveries including (though not limited to) the existence of water ice in polar craters (Lawrence et al., 2013; Paige et al., 2013; Chabot et al., 2018), the enigmatic hollows (Blewett et al., 2018), an extraordinarily chemically reduced crust (McCoy et al., 2018), the unusual geometry of the global magnetic field (Anderson et al., 2011), evidence for ancient remanent magnetization of the crust (Johnson et al., 2018), the different seasonalities of the exospheric species (McClintock et al., 2018), and an exceptionally active magnetosphere (Slavin et al., 2014, 2018; Korth et al., 2018) all raise fundamental questions about the formation and 
evolution of planets and their interactions with the space environment. Exploration and study of this end-member planet is essential for understanding planetary formation, how and why planets evolve, and the interplay of atmospheres, magnetospheres, and rocky planets when close to their host stars.

The high-priority science questions in this report reflect an ongoing conversation within the Mercury science and exploration community. These questions are collated from recent discussions at the Mercury: Current and Future Science of the Innermost Planet Workshop in (Mercury Workshop, 2018), community feedback for the white paper on landed science at Mercury, the existing body of literature, as well as direct community engagement. Through a survey (Mercury Community Survey, 2019) distributed to the planetary science community (e.g., to subscribers of the Mercury-Planet-List and the Planetary Exploration Newsletter, as well as through social media), feedback was solicited to identify the big-picture, vital science questions to be accomplished at Mercury, as well as more broadly in planetary science. In addition to direct input and open feedback, individuals were invited to rate the priority of the questions and themes listed below (Section 2). A broad cross-section of the planetary science community, disciplinary interest and otherwise, contributed to the development of these questions and themes. Using a 1-5 scale (where $1=$ low priority and $5=$ high priority), each of the items discussed below were identified as having a score of 4 or 5 by a considerable majority (i.e., $>60 \%$ with most $>75 \%$ ). More than thirty individual scientists beyond the committee that prepared this report contributed responses to the survey. The numerical results and fundamental science questions here were first reported in a whitepaper delivered to the NASA Planetary Science Division in August 2019 (Hauck et al., 2019).

The fundamental science questions listed below are all strongly synergistic with at least a majority, if not all, of their companion questions. It is clear that multiple lines of inquiry, often interdisciplinary in nature, are an essential element to understanding the complexity of planet Mercury.

\section{Fundamental Science Questions for Mercury}

\subsection{How did Mercury form?}

MESSENGER revealed much about Mercury, such as its extremely chemically reduced nature, exotic chemistry including a S- and C-rich surface and Si likely in the core, and its enormous metallic core that remains still partially molten today (e.g., Nittler et al., 2011; Klima et al. 2008; Hauck et al., 2013). All of these observations add to the fundamental fact that Mercury is unlike any of the other planets in our solar system, leading to the basic question: how did Mercury form (Ebel and Stewart, 2018)? This high-priority question is foundational to understanding the origin of Mercury and the formation of planets in our solar system.

\section{Example focused questions:}

- What were the building blocks of Mercury and how did they compare to those of the other terrestrial planets?

- What roles might giant impact(s), planet migration, or location of planetary formation have had on the structure and composition of Mercury?

- How do terrestrial bodies with high ratios of metal-to-silicate form and what are the implications for terrestrial planet formation?

- What is the bulk elemental composition of Mercury?

- What is the stable isotopic composition of Mercury?

- What does the composition of Mercury tell us about the protoplanetary disk? 


\subsection{How did Mercury differentiate and acquire its interior structure?}

Mercury's internal structure reflects both its unique metal-to-silicate ratio among the terrestrial planets and the set of processes that led to its present interior structures (e.g., Margot et al., 2018). MESSENGER found evidence of an ancient graphite flotation crust as well as a Mg-rich, Fe-poor silicate crust and mantle, overlying a very large metallic core (e.g., Nittler et al. 2018). Understanding how its starting materials transform into Mercury's current internal layering is crucial for determining the starting point of the planet's geological and internal evolution, and for how differentiation and magma oceans operate more generally.

Example focused questions:

- Why does Mercury have such a large metal-to-silicate ratio?

- What are the compositional constraints on Mercury's interior layers, such as its solid and liquid cores, mantle, and potential other layers?

- What can Mercury's crustal and lithospheric properties tell us about its thermal evolution?

- Did Mercury have a magma ocean, and, if so, how did its crystallization affect the subsequent evolution of the planet?

- What processes led to the presence of graphite and sulfides contained in the crust?

- What is the composition of the low-reflectance material on the planet, hypothesized to be the primary crust?

- When did Mercury accrete and differentiate relative to the formation of calcium-aluminumrich inclusions?

- When did Mercury's core form?

\subsection{What is the history of Mercury's magnetic field and its generation?}

Mercury's enigmatic magnetic field is unique among the rocky planets because of its relatively low strength and particular geometry (e.g., Johnson et al., 2018). The MESSENGER-based discovery of crustal magnetization in rocks older than $3.5 \mathrm{Ga}$ point to the operation of an ancient magnetic field. Characterizing Mercury's magnetic field therefore remains an essential, highpriority component of understanding both Mercury and planetary magnetic fields everywhere. It is also closely tied to understanding the planet's interior structure. Indeed, electromagnetic induction has already been used to independently confirm the outer boundary of Mercury's core (Johnson et al., 2016), and this technique could in the future provide constraints on the electrical conductivity structure of the silicate mantle and crust. Furthermore, these better-constrained core size and properties are now being incorporated into sophisticated models to simulate an accurate representation of the coupling between Mercury's core and local space environment (Jia et al., 2015).

\section{Example focused questions:}

- What is the source of Mercury's weak, axisymmetric, and hemispherically asymmetric internal magnetic field?

- What are the distribution and age(s) of crustal magnetization?

- What are the minerals that carry magnetization on Mercury?

- Has Mercury's internal field operated continuously throughout the planet's history?

- Were the geometry and strength of an ancient field different from that observed today?

- What can magnetic induction reveal about the electrical conductivity structure of the interior? 


\subsection{How do Mercury's surface and interior reflect the evolution of the planet?}

Although it is the smallest planet with the thinnest mantle and fractionally largest metallic core among the solid bodies in the solar system (e.g., Margot et al., 2018), Mercury also has an extended history of volcanism and a dominantly contractional tectonic history (e.g., Byrne et al., 2018a,b; Denevi et al., 2018). Further investigation of the planet's volcanic and tectonic properties will enhance our view of how the internal processes of cooling and mantle convection are expressed at the surface (e.g., Hauck et al., 2018). Further, Mercury's bombardment history, albeit only since $\sim 4 \mathrm{Ga}$, provides a basis for improving understanding of the bombardment history of the inner solar system (e.g., Chapman et al., 2018), and the role that major impacts have had in shaping the evolution of terrestrial planetary bodies - including on the planet's volcanic and tectonic behavior.

Example focused questions:

- How much, if any, of Mercury's present crust represents a primary crust composed of graphite?

- What does Mercury's history of volcanism indicate about the composition(s) and depth(s) of the sources of melting?

- How and why has the nature and extent of volcanism changed so dramatically through time on Mercury?

- What is the mineralogy of the various volcanic units on the planet, and how does that mineralogy inform understanding of the thermochemical evolution of Mercury?

- To what degree have secondary processes affected the surface composition and mineralogy of Mercury (e.g., smelting, space weathering, thermal transport of volatiles)?

- What is the origin of the largest physiographic unit on Mercury, the intercrater plains?

- Are geological processes such as formation of hollows and scarps actively taking place today?

- How has the bombardment history of Mercury influenced the planet's evolution?

- How and when did Mercury enter its current 3:2 spin-orbit resonance, and what role has the coupled orbital and rotational history had on the planet's evolution?

2.5. What is the nature of the complex interactions among Mercury's external drivers and the planet's magnetosphere, exosphere, surface, and interior?

Mercury is unique in that it possesses a surface-bounded exosphere with a substantial magnetosphere that shields the planet from the impinging solar wind under all but the most extreme solar conditions. Thus, Mercury exhibits a range of complex interactions that make it a prime laboratory for exploring plasma phenomena that are ubiquitous throughout the solar system and beyond. Mercury's interaction with the solar wind is similar to, but more extreme than, that of Earth, with intense solar wind driving that produces high rates of magnetopause reconnection, the formation of Kelvin-Helmholtz instabilities, and flux transfer event generation as the Sun's magnetic field piles up against the planetary obstacle on the dayside (DiBraccio et al., 2013; Gershman et al., 2013, 2015; Imber et al., 2014; Slavin et al., 2012a; Sundberg et al., 2010). This dayside coupling between the Sun and planetary magnetic fields generates a process of convecting mass, energy, and momentum throughout the magnetosphere, leading to further dynamics that are often more extreme than those observed at Earth including: particle precipitation in the magnetospheric cusps, acceleration of planetary ions (i.e., those derived from surface materials) from cusp to magnetotail flux ropes, tail loading and unloading with additional substorm-like 
activity, dipolarization events, fast plasma flows, plasma mantle formation, and more (Delcourt et al, 2003; 2013; DiBraccio et al., 2015; Imber and Slavin, 2017; Jasinski et al., 2017; Poh et al., 2016, 2018; Raines et al., 2014; Slavin et al., 2010, 2012b; Sun et al., 2016; Winslow et al, 2014;). Its magnetosphere is populated by a variety of ions of planetary origin, such as $\mathrm{Na}+, \mathrm{O}+$ and $\mathrm{He}+$ (Zurbuchen et al., 2011), primarily in the cusps and central plasma sheet. These mostly serve as tracers of magnetospheric dynamics due to their low abundance relative to protons (Raines et al., 2013; Gershman et al., 2014). Solar-related phenomena such as coronal mass ejections and solar energetic particle events create an intense response (Slavin et al., 2014) including the most extreme, which completely strips away the dayside magnetosphere allowing for the solar wind to directly interact with the planetary surface (Jia et al., 2019; Slavin et al., 2019; Winslow et al. 2020). Magnetospheric convection, particle energization, and particle precipitation are just some of the aspects that are greatly affected by Mercury's interactions with the Sun. Mercury's planetary magnetic field also responds to these external drivers through magnetic induction, contributing yet another dimension to this dynamic interaction.

Mercury's exosphere is populated by several species derived from distinct combinations of source processes related to these same external solar drivers, as well as other non-solar processes such as micrometeoroid impacts and sputtering by planetary ions. MESSENGER revealed unexpected seasonalities in the exospheres of individual species, including strong connections of $\mathrm{Ca}$ and potentially $\mathrm{Al}$ and Mn with dust from comet 2P/Encke (Burger et al., 2014; Killen and Hahn, 2015; Vervack et al., 2016), regular enhancements of Mg from connections to Mg-rich regions of the surface (Merkel et al., 2018), and a surprising repeatability in the Na exosphere from year to year, suggesting that sputtering effects may be smaller than predicted (Cassidy et al., 2015), possibly because seeing effects on terrestrial observations have been misinterpreted (Killen, 2020). Theoretical and laboratory studies have suggested that planetary ions originate not only through photoionization of neutral exospheric material but that direct release of ions to the magnetosphere is possible (e.g., Berezhnoy, 2018; McLain et al., 2011), opening potential new pathways for interactions among the surface, exosphere, and magnetosphere. Mercury provides unique opportunities to study these processes by understanding how the exosphere is generated and maintained, as well as how the exosphere and magnetosphere respond to one another.

\section{Example focused questions:}

- How does the continual bombardment of the surface by various solar and non-solar drivers and the escape of material from the system via the exosphere and magnetosphere affect the long-term evolution of the surface composition (i.e., space weathering)?

- How is material driven from the surface by solar wind and interplanetary dust particles, as well as by solar radiation and regular cometary dust stream encounters?

- How does material that returns to the surface affect the distribution of volatiles?

- How does neutral exospheric material that is ionized and driven back to the surface by the magnetosphere provide a feedback for the generation of the exosphere?

- How does Mercury's global magnetic field protect the dayside planetary surface from direct solar wind impact during periods of extremely high solar wind pressure and intense southward interplanetary magnetic field?

- What are the geometry and dynamics of the high-latitude dayside cusp regions on Mercury and how do they relate to the interaction between the solar wind and Mercury's dayside magnetosphere via dayside magnetic reconnection and the total magnetic flux transfer to the nightside magnetosphere? 
- What controls the generation of field-aligned currents at Mercury, and does the same current closure mechanism applies to the nightside substorm current wedge?

- What are the causes of observed asymmetries, including plasma and magnetic field properties, substorm signatures, and magnetic reconnection in Mercury's magnetotail?

\subsection{What are the origin, history, and inventory of Mercury's volatiles?}

The highly chemically reducing nature of Mercury, as well as its surface enrichment in volatile elements, has led to a complex and exotic thermal and magmatic evolution of the planet (e.g., Nittler et al., 2018). Mercury's surface features indicate past explosive volcanism, consistent with the presence of magmatic volatiles in its interior. Furthermore, the permanently shadowed craters near Mercury's poles show evidence for extensive water ice and organic-rich volatiles exposed at the surface (e.g. Chabot et al., 2018), providing a tantalizing target for in situ scientific exploration. Understanding the origin of Mercury's volatiles will provide key insight into the sources and distributions of volatiles across the inner solar system and the availability more broadly of volatiles for terrestrial planetary growth. In addition, advancements towards understanding Mercury's volatile record will place tighter constraints on the planet's formation and differentiation history, as well as on the composition of its interior, furthering our knowledge of how Mercury acquired its current interior structure.

\section{Example focused questions:}

- What role did volatiles play in melting of the interior, the ascent and transport of magmas, and on the styles of surface eruptions?

- Do alkali abundances on Mercury's surface reflect magmatic abundances or were these materials thermally redistributed?

- What is the reason for Mercury's low oxygen fugacity, and did volatiles play a role in its origin?

- What is the source(s) of the polar deposits?

- What is the origin of the hollows?

- What can the exosphere tell us about the redistribution of volatiles over time?

- What is the total inventory of volatiles on Mercury, and what do the volatile abundances tell us about their distribution in the inner solar system and within the protoplanetary disk?

\section{Recommendations and Conclusions}

Continued exploration of the innermost planet is essential to provide fundamental insights into Mercury, the other planets in the solar system and beyond. Answering the questions listed here will also enhance comparative planetology studies by providing, for example, information regarding lateral heterogeneities with distance from the Sun, space weathering effects on airless bodies, the behavior of elements at extreme conditions, and the origin and distribution of volatiles in the inner solar system and on exoplanets and their satellites orbiting close to their host star. It is crystal clear how vital it is that future studies focus on how multiple disciplines and modes of inquiry can contribute synergistically to a better understanding of this enigmatic planet. Further, to fully address such multifaceted and multidisciplinary questions, the community of planetary science must be one that is truly interdisciplinary, diverse, equitable, inclusive, and accessible. The continued exploration of Mercury, well beyond the ESA/JAXA BepiColombo mission, should be completed through a rich set of research and analysis opportunities, ground-based observations, and exploration missions - including landed missions (Byrne et al., 2020) - to fully leverage Mercury as the key to understanding the characteristics of rocky planets close to their host stars. 
Anderson, BJ, et al., (2011), Science, 10.1126/science.1211001. * Berezhnoy, AA (2018), Icarus, 10.1016/j.icarus.2017.08.034. - Blewett, DT, et al. (2018) Ch. 12 in Mercury: The View After MESSENGER.. — Burger, MH, et al. (2014), Icarus, 10.1016/j.icarus.2014.04.049. — Byrne, $\mathrm{PK}$, et al (2020) White paper on the case for landed Mercury science https://tinyurl.com/MercuryLanderWhitePaper. - Byrne, PK, et al. (2018a) Ch. 10 in Mercury: The View After MESSENGER. - Byrne, PK, et al. (2018b) Ch. 11 in Mercury: The View After MESSENGER. - Cassidy, TA, et al. (2015), Icarus, 10.1016/j.icarus.2014.10.037. - Chabot, NL, et al. (2018) Ch. 13 in Mercury: The View After MESSENGER. - Chapman, CR, et al. (2018) Ch. 9 in Mercury: The View After MESSENGER. - Denevi BW, et al. (2018) Ch. 6 in Mercury: The View After MESSENGER. - DiBraccio, GK et al. (2013) JGR: Space Phys, 10.1002/jgra.50123 - DiBraccio, GA, et al. (2015) Planet. Space Sci., 115, 10.1016/j.pss.2014.12.016 Ebel, DS and ST Stewart (2018) Ch. 18 in Mercury: The View After MESSENGER a Gershman, DJ, et al. (2013) JGR:Space Phys, 10.1002/2013JA019244 @ Gershman, DJ, et al. (2014), JGR:Space Phys 10.1002/2014GL060468 Gershman, DJ, et al. (2015) JGR:Space Phys, 10.1002/2014JA020903 n Hauck, SA, II, et al. (2013) JGR:Planets, 10.1002/jgre.20091. @ Hauck, SA, II, et al. (2018) Ch. 19 in Mercury: The View After MESSENGER. - Hauck, SA, II, et al. (2019) https://www.lpi.usra.edu/NASA-academies-resources/Mercury-high-priority-questions final.pdf, - Imber, SM, et al. (2014) JGR:Space Phys, 10.1002/2014JA019884 - Imber, SM, \& Slavin, JA (2017). JGR:Space Phys, 10.1002/2017JA024332 - Jasinski, JM, et al, (2017) JGR:Space Phys, 10.1002/2017JA024594 @ Jia, X, et al. (2015) JGR:Space Phys, 10.1002/2015JA021143 - Jia, X, et al. (2019) JGR:Space Phys, 10.1029/2018JA026166, Johnson, CL, et al. (2018) Ch. 5 in Mercury: The View After MESSENGER. - Johnson, CL, (2016) GRL, 10.1002/2015GL067370. - Killen, RM (2020), EPSC Abstracts, 14, EPSC2020-3. - Killen, RM, and JM Hahn (2015), Icarus, 10.1016/j.icarus.2014.11.035. Klima, RL, et al (2018) GRL 10.1002/2018GL077544 - Korth, H, et al. (2018) Ch. 16 in Mercury: The View After MESSENGER. — Lawrence, DJ, et al. (2013) Science, 10.1126/science.1229953. - McLain, JL, et al. (2011), JGR:Planets, 10.1029/2010JE003714. - Mercury Community Survey, July-August (2019) - Mercury: Current and Future Science of the Innermost Planet Workshop (2018) Merkel, AW, et al. (2018), GRL,10.1029/2018GL078407. Nittler, LR, et al. (2011) Science 10.1126/science.1211567 Nittler, LR, et al. (2018) Ch. 2 in Mercury: The View After MESSENGER. - Margot, JL, et al. (2018) Ch. 4 in Mercury: The View After MESSENGER. McClintock, WE, et al. (2018) Ch. 14 in Mercury: The View After MESSENGER. - McCoy, TJ, et al. (2018) Ch. 7 in Mercury: The View After MESSENGER. - Paige, DA, et al. (2013) Science 10.1126/science.1231106. — Poh, G, et al. (2016), 10.1002/2016JA022552. — Poh, G, et al (2018) GRL, 10.1029/2018GL080601. - Raines, JM, et al., 10.1029/2012JA018073. * Raines, JM, et al. (2014), 10.1002/2014JA020120. - Slavin, JA, et al. (2010) Science, 10.1126/science.1188067 Slavin, JA, et al. (2012a) JGR: Space Phys, 10.1029/2012JA017926 - Slavin, JA, et al. (2012b) JGR: Space Phys, 10.1029/2011JA016900 - Slavin, JA, et al. (2014), JGR: Space Phys, 10.1002/2014JA020319. - Slavin, JA, et al. (2018) Ch. 14 in Mercury: The View After MESSENGER. - Slavin, JA, et al. (2019), JGR: Space Phys, 10.1029/2019JA026892. - Sun, WJ, et al. (2016), JGR: Space Phys, 10.1002/2016JA022787. " Sundberg, T, et al. (2012), JGR: Space Phys, 10.1029/2011JA017268. @ Vervack, RJ, Jr, et al. (2016), GRL, 10.1002/2016GL071284. - Winslow, RM, et al. (2014), 10.1002/2014GL060258.

Winslow, RM, et al. (2020), ApJ, 10.3847/1538-4357/ab6170 — Zurbuchen, TH, et al. (2011), Science, 10.1126/Science.1211302. 